\title{
Factors Affecting Quality of Life in Patients of Cancer Cervix
}

Prof Uma Singh, Dr Z Rahman, Dr Manjulata Verma, DrS Qureshi, Dr A Nischal

K G Medical university, Lucknow, India

\section{Introduction}

- $1,22,844$ women are diagnosed with carcinoma cervix in India every year

- Advances in treatment and availability of facilities has improved the overall survival

- Time has arrived to also focus on quality of life (QOL) in these patients

- Treatment should include giving them good quality of life

\section{Aim of the study}

- To assess the QOL in women suffering from cancer cervix before \& after treatment

- To study the factors affecting the QOL in these women

\section{QOL - WHO definition}

The way an individual perceives his or her own position in the context of culture and system of values in which he or she exits together with own aims, limitations, standards an concerns.

\section{Questionnaire based face to face interview}

- $\quad$ Before treatment

- 3 months after treatment

- 6 months after treatment

\section{Material and Methods}

- Prospective cohort study over 1year

- $\quad$ Dept. of Obst and Gynae and Radiotherapy

King George's Medical University, Lucknow, India

- Institutional ethical committee approval obtained

- Written informed consent taken

Questionnaire used

- General cancer QOL- EORTC QLQ C30

- Specific cancer cervix- EORTC QLQ Cx24

\section{Independent factors studied}

- Age in years - Menstrual status

- Marital status - Tobacco use

- Education - Degree of

- Residence differentiation of tumor

- Histology

- Treatment modality
- Income - Stage of cancer

- Tumor size

\begin{tabular}{l|l|c|c|c|c|c|}
\hline $\begin{array}{l}\text { Independent } \\
\text { variables }\end{array}$ & \multicolumn{2}{|l|}{ Global health } & \multicolumn{2}{l|}{$\begin{array}{l}\text { Total functional } \\
\text { scale }\end{array}$} & \multicolumn{2}{l|}{$\begin{array}{l}\text { Total symptoms } \\
\text { scale }\end{array}$} \\
\cline { 2 - 7 } & $\beta$ coefficient & $\begin{array}{c}\mathrm{p}- \\
\text { value }\end{array}$ & $\begin{array}{c}\beta \\
\text { coefficie } \\
\mathrm{nt}\end{array}$ & $\begin{array}{c}\mathrm{p}- \\
\text { value }\end{array}$ & $\begin{array}{c}\beta \\
\text { coefficien } \\
\mathrm{t}\end{array}$ & $\begin{array}{c}\mathrm{p}- \\
\text { value }\end{array}$ \\
\hline Income & 1.11 & 0.21 & 1.02 & 0.56 & 1.23 & 0.11 \\
\hline Education & 2.34 & $0.03^{*}$ & 2.38 & $0.01^{*}$ & 2.23 & $0.03^{*}$ \\
\hline Tobacco use & 0.99 & $0.02^{*}$ & 0.87 & $0.04^{*}$ & 0.47 & $0.02^{*}$ \\
\hline $\begin{array}{l}\text { Degree of } \\
\text { differentiation }\end{array}$ & 2.45 & $0.01^{*}$ & 2.98 & $\begin{array}{c}0.002 \\
*\end{array}$ & 2.34 & $0.02^{*}$ \\
\hline Tumor size & 2.21 & $0.02^{*}$ & 3.33 & $\begin{array}{c}0.002 \\
*\end{array}$ & 2.56 & $0.03^{*}$ \\
\hline
\end{tabular}

a months $\quad 3$ months $\square$ Baseline
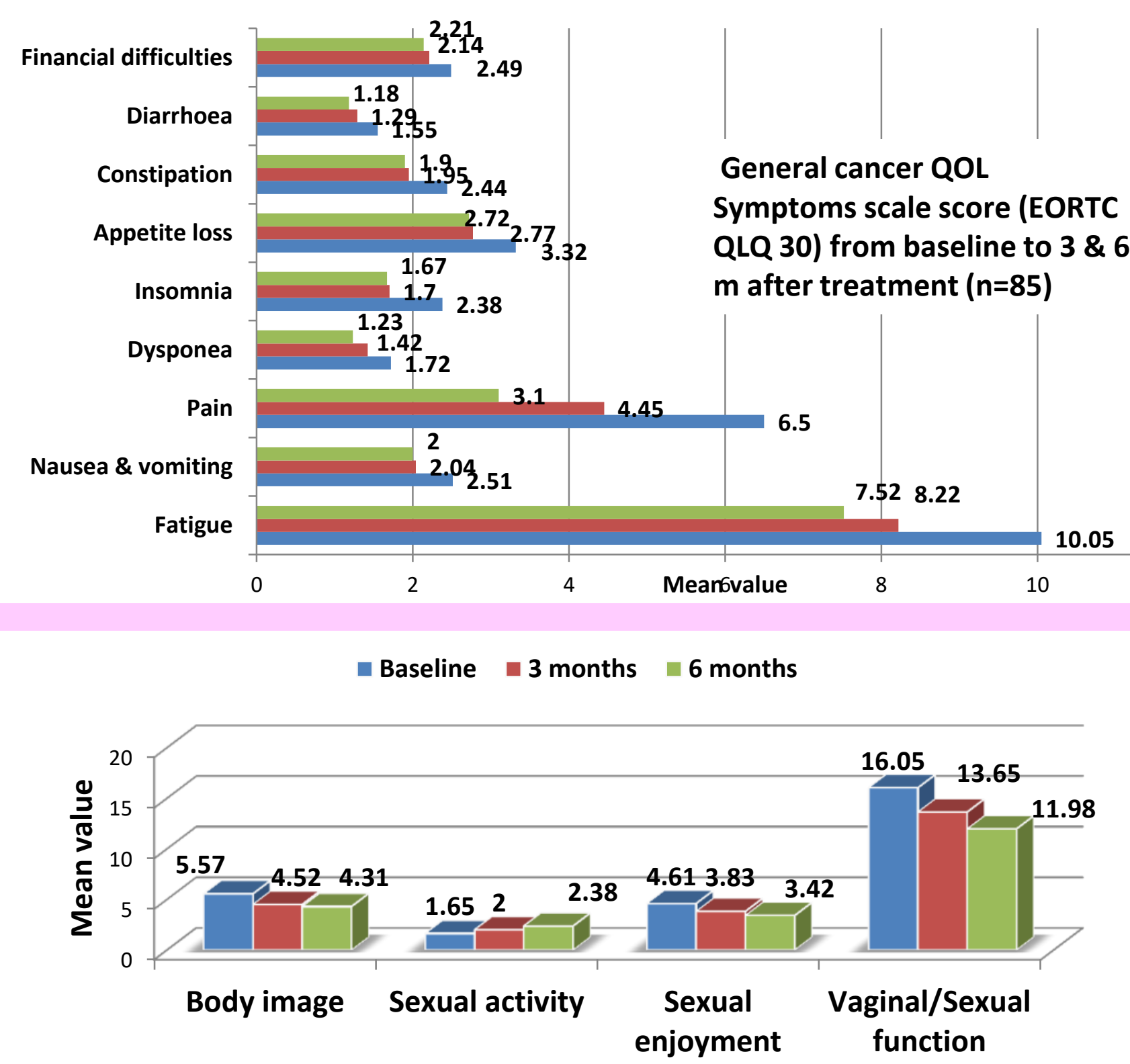

CaCx specific QOL Functional scale score (EORTC QLQ Cx 24) from baseline to 3 months and 6 months after treatment $(n=85)$

\section{Factors affecting QOL}

Multivariate regression analysis

- Education

- Degree of differentiation of tumor

- Size of tumor

- Type of treatment
- Tobacco use

\section{Conclusions}

- Role function and cognitive function - improvement but not statistically significant

- Emotional functions - significant improvement

- Social functioning - no improvement

- Physical symptoms - variable response

- Sexual function-marginal improvement

\begin{tabular}{|c|c|}
\hline $\begin{array}{l}\text { Timing of } \\
\text { evaluation }\end{array}$ & $\begin{array}{l}\text { Global health } \\
\text { (Mean } \pm \text { SD) }\end{array}$ \\
\hline \begin{tabular}{|l} 
Baseline \\
\end{tabular} & $4.91 \pm 1.86$ \\
\hline 3 months & $5.44 \pm 1.79$ \\
\hline $\begin{array}{l}6 \text { months } \\
\end{array}$ & $5.69 \pm 2.19$ \\
\hline $\mid p$-value ${ }^{1}$ & \\
\hline $\begin{array}{l}\text { Baseline to } 3 \\
\text { months }\end{array}$ & 0.09 \\
\hline \begin{tabular}{|l|} 
Baseline to 6 \\
months
\end{tabular} & $0.04^{*}$ \\
\hline
\end{tabular}

Baseline 3 months 6 months

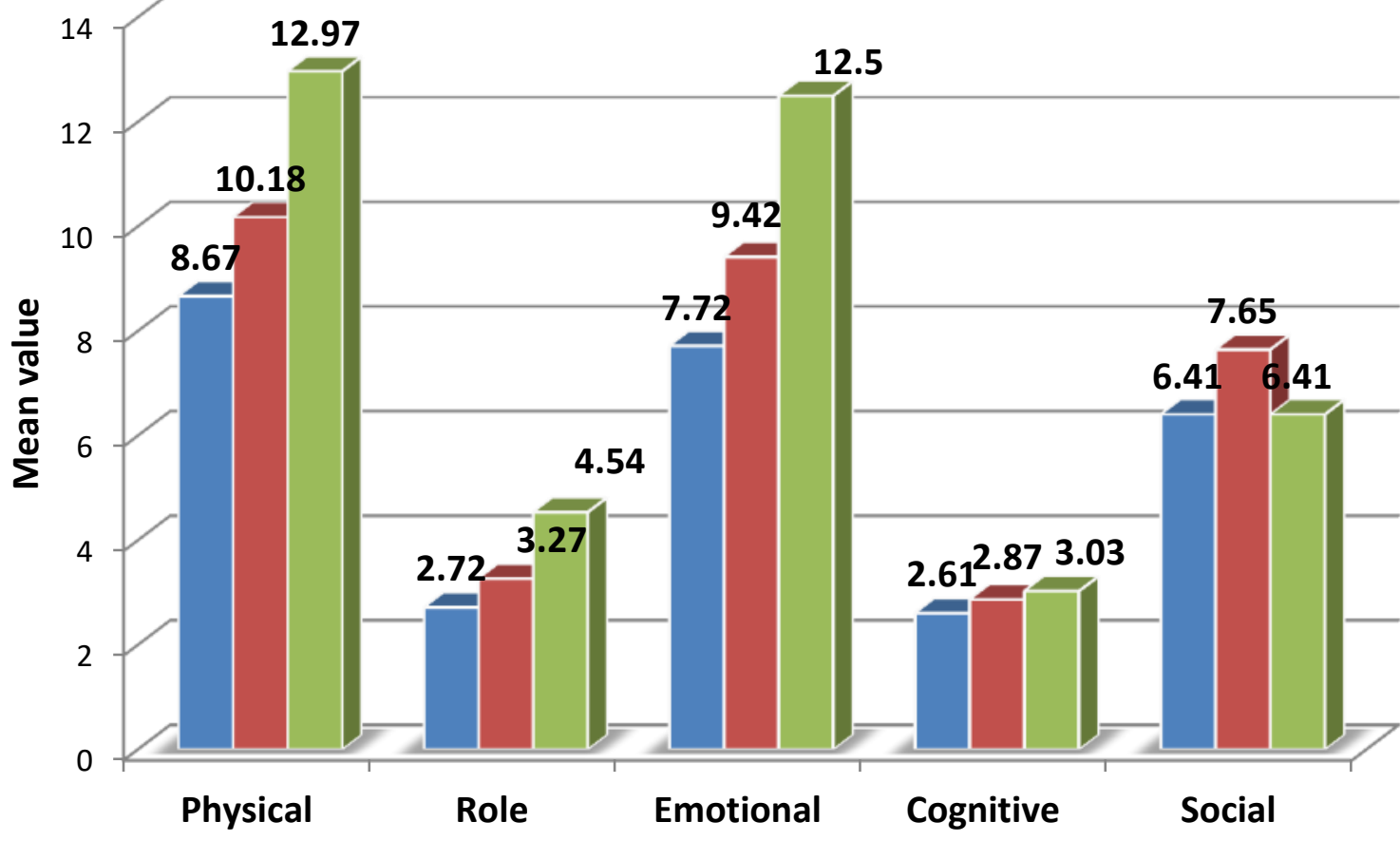

General cancer QOL Functional scale score (EORTC QLQ30) from baseline to $3 \& 6$ months after treatment $(n=85)$

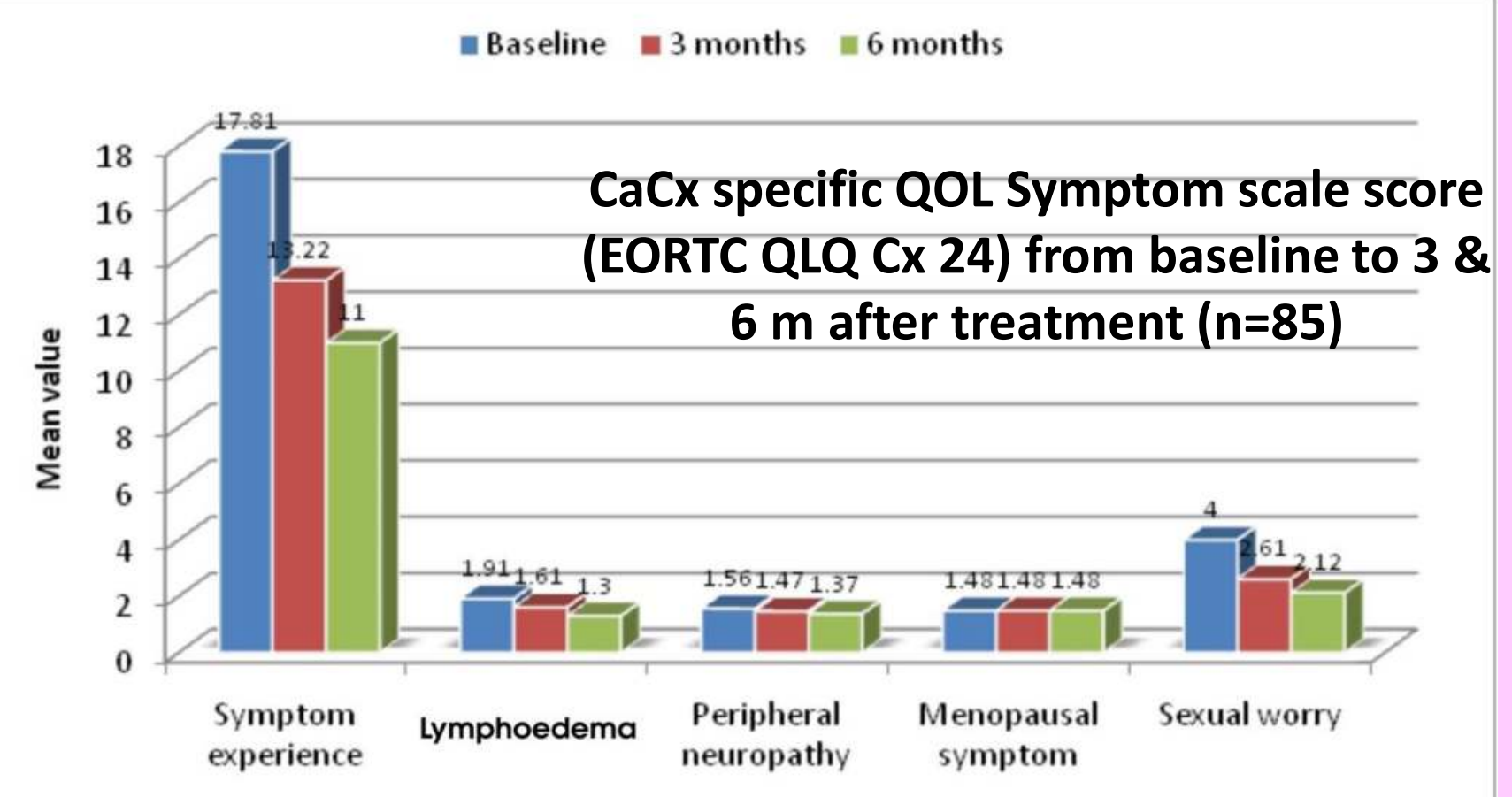

\section{Key message}

Management of malignancy should not be limited to only treating the clinical disease but it should also involve the plan which can give the overall best quality of life (QOL) 